$30 \%$ within the not negative groups (excluding SADS where it was $100 \%)$. Factors contributing to low rates of genetic testing and phenotypic predictors of positive genotype warrant further investigation.

\title{
Moderated posters
}

\section{REAL-LIFE USE OF IMPELLA ${ }^{\circledR}$ CP: THE NORTHERN IRISH EXPERIENCE}

U Azhar Khan, M Saad Ahmed, J Conleth Murphy. Royal Victoria Hospital, Belfast, UK

\subsection{6/heartjn-2021-ICS.23}

Introduction The Impella ${ }^{\circledR}$ CP (ABIOMED Inc., Danvers MA, USA) is a relatively novel percutaneously inserted mechanical circulatory support (MCS) system. It is a $14 \mathrm{Fr}$ micro-axial temporary left ventricular assist device, which provides continuous forward flow of up to $4.3 \mathrm{~L} / \mathrm{min}$ into the ascending aorta to augment cardiac output. It is indicated to provide additional haemodynamic support in cases of advanced cardiogenic shock (CS), primarily in acute myocardial infarction (AMI). It is also used in selected high-risk patients undergoing complex percutaneous coronary intervention (PCI). It is a widely known modality but data from randomized controlled trials (RCT's) have not demonstrated a clear clinical benefit. We aim to describe our experience with use of Impella ${ }^{\circledR} \mathrm{CP}$ in our institution.

Methods The Belfast Health \& Social Care Trust is a high-volume interventional centre, which performs approximately 2500-3000 PCI per annum, of which 650 are emergency procedures. We retrospectively reviewed all patients who were treated with Impella ${ }^{\circledR} \mathrm{CP}$ for cardiogenic shock after acute myocardial infarction as well as those undergoing elective high-risk PCI [table 1]. We report patient demographics,

\begin{tabular}{|c|c|c|c|}
\hline & Total cases & $\begin{array}{c}\text { Acute MI with } \\
\text { Cardiogenic Shock }\end{array}$ & Elective High-risk PCl \\
\hline Number of patients & 7 & 5 & 2 \\
\hline Age (years) (Range) & $56.4(37-76)$ & $49.8(37-68)$ & $73(70-76)$ \\
\hline Male sex, $n(\%)$ & $7(100)$ & $5(100)$ & $2(100)$ \\
\hline Hypertension, $\mathrm{n}(\%)$ & $7(100)$ & $1(20)$ & $2(100)$ \\
\hline Dyslipidemia, $n(\%)$ & $3(43)$ & $1(20)$ & $2(100)$ \\
\hline Diabetes Mellitus, $n$ (\%) & $2(28)$ & $0(0)$ & $2(100)$ \\
\hline Ischemic heart disease, $n(\%)$ & 1(14) & $0(0)$ & $1(50)$ \\
\hline \multicolumn{4}{|l|}{ Procedure-related complications } \\
\hline Limb Ischemia, n (\%) & $2(28)$ & $2(40)$ & $0(0)$ \\
\hline $\begin{array}{l}\text { Bleeding requiring transfusion, } n \\
(\%)\end{array}$ & $2(28)$ & $1(20)$ & $1(50)$ \\
\hline \multicolumn{4}{|l|}{ Before device placement } \\
\hline $\begin{array}{l}\text { Intra-aortic balloon pump before } \\
\text { Impella placement, } n(\%)\end{array}$ & $5(72)$ & $5(100)$ & $0(0)$ \\
\hline $\begin{array}{l}\text { Lactate }(\mathrm{mmol} / \mathrm{L}) \text {, median } \\
\left(\mathrm{Q} 1, \mathrm{Q}_{3}\right)\end{array}$ & & $6.2(2.6,6.7)$ & - \\
\hline Arterial pH, median $\left(\mathrm{Q}_{1}, \mathrm{Q}_{3}\right)$ & & $7.18(7.15,7.22)$ & - \\
\hline \multicolumn{4}{|l|}{$\begin{array}{l}\text { In-hospital treatment \& } \\
\text { outcome }\end{array}$} \\
\hline Surgical LVAD placement, $n(\%)$ & $1(14)$ & $1(20)$ & $0(0)$ \\
\hline $\begin{array}{l}\text { Extracorporeal membrane } \\
\text { oxygenation (ECMO), } n(\%)\end{array}$ & $2(28)$ & $2(28)$ & $0(0)$ \\
\hline $\begin{array}{l}\text { Length of stay in survivors (days), } \\
\text { mean (Range) }\end{array}$ & $70.2(5-185)$ & $110.3(55-185)$ & $10(5-15)$ \\
\hline 30-day mortality, $n(\%)$ & $2(28)$ & $2(40)$ & $0(0)$ \\
\hline
\end{tabular}

Abstract 23 Table 1 Baseline characteristics, procedure-related complications, in-hospital treatment and outcomes of all impella cases including patients with acute myocardial infarction (MI)/ cardiogenic shock and elective high-risk percutaneous coronary intervention $(P C)$

device-related complications and clinical outcomes in these cohorts.

Results Since March 2018, 7 patients have received Impella ${ }^{\circledR}$ CP support. 5 patients presented with AMI complicated by CS, and the remainder 2 had elective high-risk PCI. Mean age in the CS cohort was 49.8 years, with an average of one case every 232 days. Mean age in the elective high-risk PCI cohort was 73 years. In the CS cohort, 3 patients were subsequently transferred to a quaternary transplant centre with subsequent advanced heart failure (HF) input (2 extracorporeal membrane oxygenation and 1 surgical left ventricular assist device). 1 CS patient was transferred but did not undergo advanced HF input and was palliated. 1 CS patient died in the cathlab. In the total cohort $4 / 7$ (56\%) of patients had direct devicerelated complications (2/7 limb ischaemia, 2/7 bleeding requiring blood transfusions). Overall 30-day mortality in the CS cohort was $40 \%(2 / 5)$.

Conclusion Impella ${ }^{\circledR} \mathrm{CP}$ use in our high-volume center is infrequent and carries a high complication rate. Overall mortality rate in our CS group remains high but it is difficult to make definitive conclusions on small numbers. Our complication rate is significant, but similar to previous documented series in higher-volume centres. Impella ${ }^{\circledR} \mathrm{CP}$ may have clinical benefits in carefully selected patients, but in the absence of data from robust multi-centre RCT's patient selection will remain speculative. The exact role for Impella ${ }^{\circledR} \mathrm{CP}$ remains unclear.

\section{SHOCKWAVE INTRAVASCULAR LITHOTRIPSY AS AN ADJUNCT TO PERCUTANEOUS CORONARY INTERVENTION IN CALCIFIED CORONARY LESIONS: A 2 YEAR, SINGLE CENTRE EXPERIENCE}

H Muragagha, K Millar, B Wei Soh, I Ullah, T Hennessy, S Arnous, T Kiernan. University Hospital Limerick, Ireland

\subsection{6/heartjnl-2021-ICS.24}

Background Calcified coronary lesions are attributed to multiple risk factors such as increasing age, smoking, DM, and CKD. PCI of calcified lesions is challenging due to impaired stent crossing, under-expansion, and mal-apposition. Shockwave intravascular lithotripsy (S-IVL) is a novel angioplasty balloon that emits sonic pulsations, thus disrupting intra-coronary calcium and often obviates the need for conventional rotational atherectomy (RA). There is limited real-world data available on the use of S-IVL and we aim to review its use in our center.

Methods A retrospective single-center cohort study was performed of all S-IVL cases between Sep 2018 and Apr 2021.

Results Thirty-seven S-IVL procedures were performed during the period of the study. The mean age is 72.32 years (range 65-87, Median age 74).76\% were male. All patients had moderate to severely calcified coronary artery lesions. Indications for PCI included: 24 unstable angina, 9 NSTEMI, 3 Stable CAD, 1 ST-elevation MI, and 2 recurrent ISR despite drugeluting balloon therapy Risk factors included: $11(29.7 \%) \mathrm{DM}$, 34(91.8\%) HTN, 32(86.4\%) dyslipidaemia, 9(24.3\%) active smoking, 7(18.9\%) CKD, 2(5.4\%) previous TIA, 3(8.1\%) previous CABG and $13(35.1 \%)$ previous PCI. All lesions were post-dilated, mainly by using a $3.5 \mathrm{~mm}$ diameter; high pressure was also applied in almost $90 \%$ of post-dilation balloons. IVUS was used in 5 cases (mainly in LM procedure) to 
optimize therapy. In 2 cases, there was a rupture of the shockwave balloon, and this was believed to be due to inadequate balloon preparation, the procedure continued with a replacement shockwave balloon without any complication. Baseline characteristics are described in table 1.

Discussion During this study, 2 episodes of hypotension were recorded during the procedures, one recovered immediately with the challenge of normal saline, the second patient who was admitted as ACS (NSTEMI), had a heavy calcified left main disease that required bifurcation stenting, Intra-aortic balloon was required for 24 hours. The patient was discharged

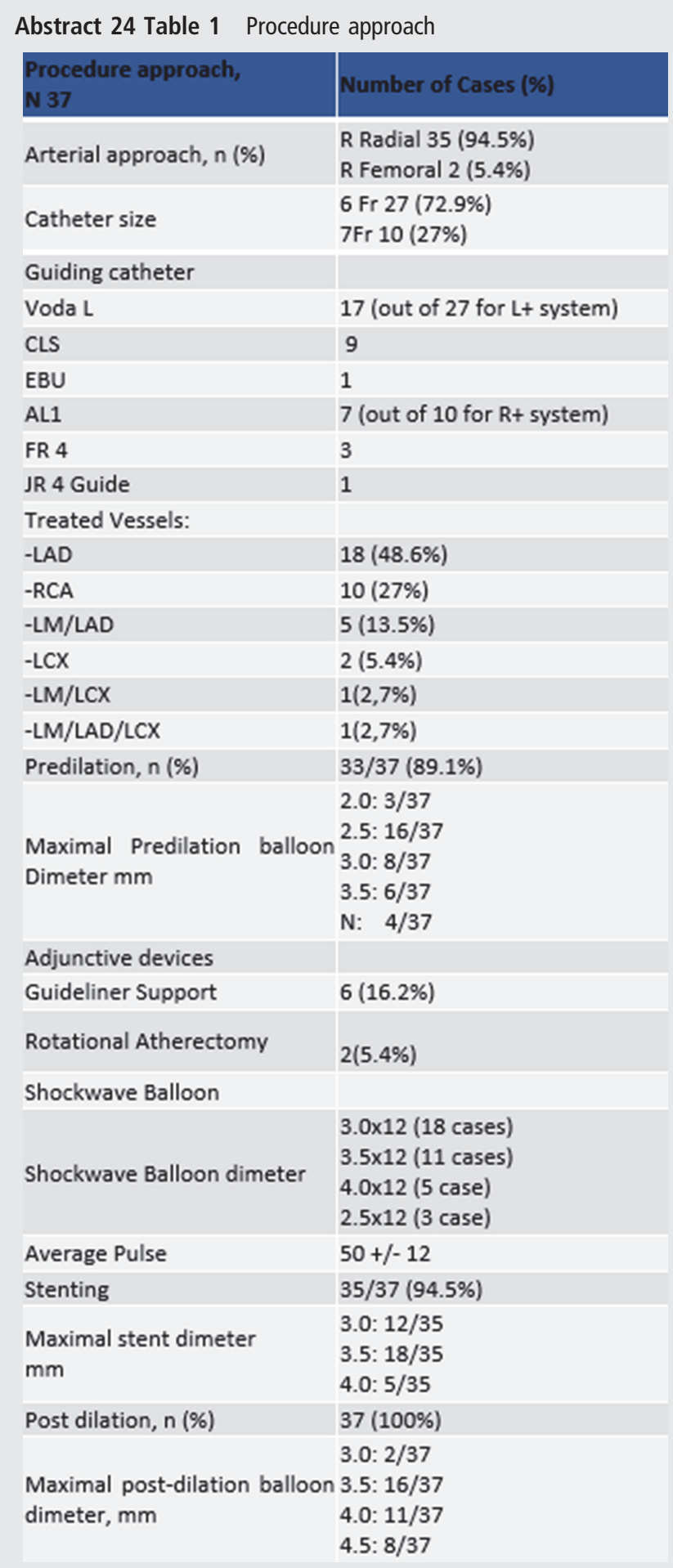

home after 7 days. Two years' follow-up showed 4 cases required to repeat coronary angiography; One case atypical chest pain with the non-obstructive disease, the second case had new lesions in different coronary arteries. The third; with prior history of CABG and stent to the native RCA with ISR had IVUS guided NC ballooning. The last one also prior to CABG, had severe ISR of his native LM/LCX even after highpressure balloon and DEB treatment. The patient was referred for Laser ablation \pm Re-do CABG. Unfortunately, due to comorbidities this patient was not fit for these procedures and passed away after two months.

Conclusion Initial experience with intravascular lithotripsy has demonstrated good angiographic outcomes without significant complications even after stent implantation. S-IVL has a minimal learning curve and has been quickly adopted as a safe and successful alternative to Rotablation. If shockwaves fail Rotablation still remains an option (2 of our cases). Patients with a previous history of CABG had a lower success rate reflective of the chronicity and complexity of the disease.

\section{CT CORONARY ANGIOGRAPHY AND COVID-19: INPATIENT USE IN ACUTE CHEST PAIN SERVICE}

M Cronin, P Wheen, R Armstrong, R Kumar, A McMahon, M White, N Sheehy, G McMahon, R Murphy. St. Lukes Hospital Kilkenny, Ireland

\subsection{6/heartjnl-2021-ICS.25}

Introduction CT coronary angiography (CTCA) is a well-validated clinical tool in the evaluation of chest pain. In January 2020 a care pathway was agreed between the cardiology, radiology and emergency departments in our facility to implement a strategy of CTCA as a first line inpatient examination for selected patients with chest pain of low to moderate risk of significant coronary artery disease. Impact on length of stay and clinical outcomes including subsequent invasive angiography and revascularisation were evaluated, including major adverse cardiac events at 30 days.

Methods Study design was retrospective. Patients referred for cardiology review between October 2019 and May 2020 with chest pain and/or dyspnoea were broken into three cohorts: a baseline cohort, a cohort with increased CTCA availability and a cohort with increased CTCA availability, but after the national lockdown due to COVID-19 in Ireland. These patients were identified via the Hospital Inpatient Enquiry dataset and correlated with chart review. Statistical analysis

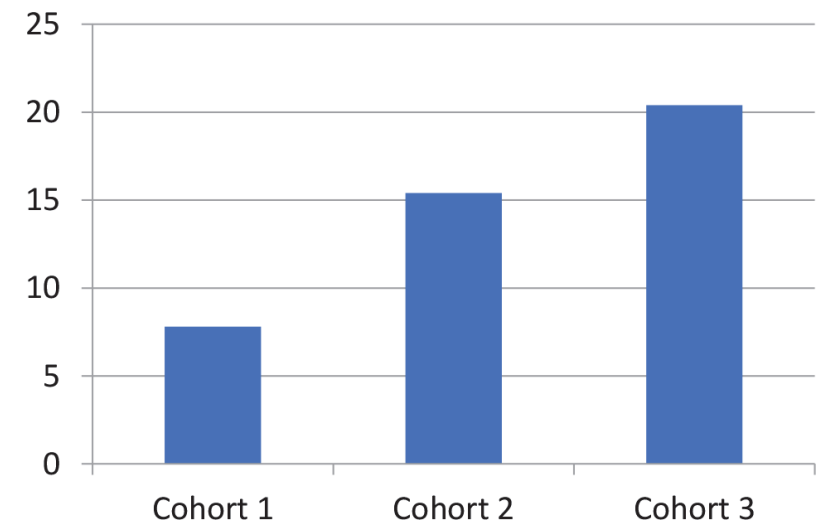

Abstract 25 Figure 1 Number of CTCA (\% of performance in overall cohort) 